\title{
PEOX-based Polymer Encapsulated Paclitaxel FID-007
}

National Cancer Institute

\section{Source}

National Cancer Institute. PEOX-based Polymer Encapsulated Paclitaxel FID-007. NCI

Thesaurus. Code C148635.

A nanoparticle-based formulation composed of the poorly water-soluble paclitaxel encapsulated within branched polymers composed of polyethyloxazoline (PEOX), with potential antineoplastic activity. Upon injection of the PEOX-based polymer encapsulated paclitaxel FID-007, the nanoparticles accumulate at the tumor site, due to the unique characteristics of the tumor vasculature, while avoiding normal, healthy tissue. Once the paclitaxel is released, it binds to tubulin inside tumor cells and inhibits the disassemblyassembly dynamics of microtubules, resulting in cell cycle arrest and cell death.

Compared to the administration of paclitaxel alone, this formulation increases paclitaxel's solubility and enhances its tumor tissue penetration and efficacy, while avoiding systemic exposure, which minimizes its toxicity. 